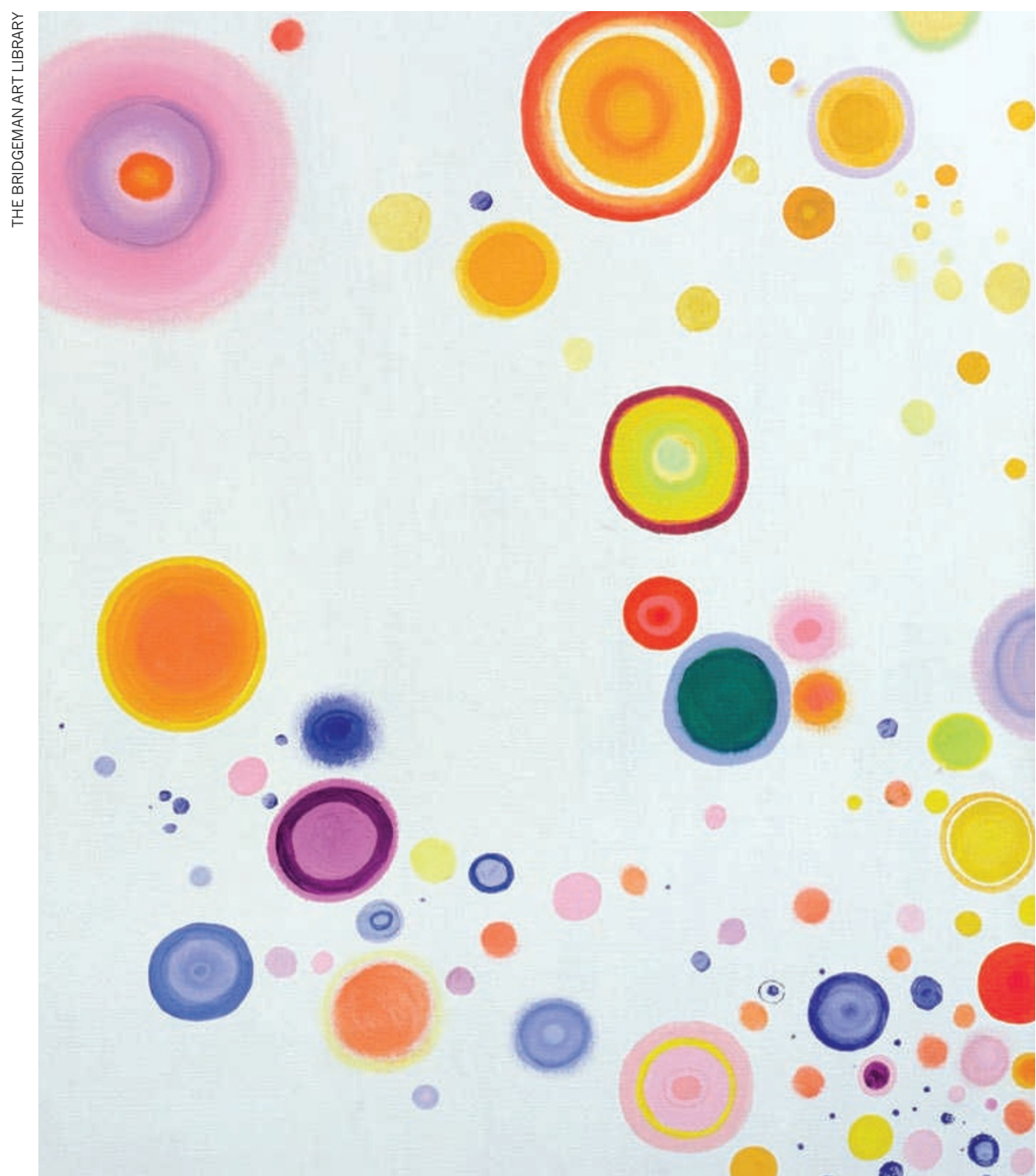

Izabella Godlewska de Aranda's painting Cosmic Joy! (2009) hints at the idea of many universes.

\title{
The untestable multiverse
}

\section{George Ellis reminds us that Brian Greene's beguiling book on parallel worlds is more theory than fact.}

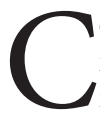

osmology must seem odd to scientists in other fields. More and more accurate data about the distant Universe are being generated by high-tech observational techniques, giving rise to an era of 'precision cosmology' - but to explain these impressive data, cosmologists are increasingly turning to untestable theories.

In The Hidden Reality, theoretical physicist Brian Greene explores one of the strangest proposals: that we live in a multiverse.
This fashionable concept supposes that large, perhaps infinite, numbers of separate universes exist in parallel to ours. Using well-constructed analogies, Greene explains nine different multiverse proposals, ranging from simple extrapolations of cosmological models to those based on quantum field theory, string theory and pure logic.

Greene carefully sets out the reasoning behind each proposal and the grounds for why it might be true. His arguments are absorbing

and convincing on their own terms. But they inexorably lead us farther from the gold standard of testability that is the scientific method. The beguiling text moves beyond established science into philosophical speculation.

Greene's nine types of multiverse are as follows. First, if space extends forever, an infinite number of domains similar to ours might lie beyond the part of the Universe that we can see. Second, some versions of inflationary theory - the idea that the newborn Universe had a fleeting period of super-fast accelerating expansion - predict the existence of innumerable other universes, with different characteristics from our own. Third, string theory, the pre-eminent theory of quantum gravity, suggests that our Universe might be one of many four-dimensional 'braneworlds' floating in a higher-dimensional space-time.

This option is developed further in the fourth and fifth proposals, which involve cyclic universes, or variations on physical parameters that are possible in the stringtheory landscape. The sixth is a quantum mechanics idea that many worlds simultaneously exist as branches of the wave function of the Universe. The seventh suggests that the Universe is a holographic projection. The eighth states that we live in one of a set of artificial universes created as simulations on a super-advanced computer. The ninth argues that it is a philosophical necessity that every possible universe must be realized somewhere, in "the grandest of all multiverses".

By presenting this plethora of theories, Greene gives the impression that the multiverse is on a sound scientific footing, but these nine arguments are mutually exclusive. We do not know how to test which is right, if any, because we cannot make direct observations of domains beyond the observational horizon - the greatest distance that light can have travelled towards us since the Universe became transparent to radiation 300,000 years after the Big Bang. Given this lack of evidence, there is a viable tenth option: that there is no multiverse at all.

Greene cites indirect evidence to support the multiverse idea. The values of the physical parameters seem to be fine-tuned to allow

$\rightarrow$ NATURE.COM For Hawking on the multiverse, see: go.nature.com/zhegpz life. For example, if the strength of the cosmological constant currently causing the accelerated expansion 
of the Universe - was much different, then galaxies would not exist and we would not be here to make measurements. Similarly, the strength of the strong nuclear force permits atoms, and hence humans, to exist. Such anthropic reasoning invokes multiverses where there is some likelihood that physical constants take on different values in each.

But probabilistic arguments only make sense if these parallel universes actually exist. And logic cannot prove their existence. For instance, a multiverse model may predict a likely value of the cosmological constant, but the reverse is not true. A particular measurement of the cosmological constant does not require a multiverse. Nor can the multiverse concept be disproved by any specific observationally determined value of the cosmological constant, for multiverses can accommodate any value. These arguments can only provide probabilistic consistency tests for some kinds of multiverse.

So one can motivate multiverse hypotheses as plausible, but they are not observationally or experimentally testable - and never will be. It is easy to support your favourite model over others because no one can prove you wrong - you can simply adjust its parameters to fit the latest information. If the Universe is a simulation (option eight), then anything is possible. However, the existence of a computer allowing such a simulation is not remotely feasible. Scientists are beginning to confuse science with science fiction.

Greene, to his credit, devotes a chapter to the question of whether the multiverse idea is a scientific theory or not. He believes it is, and even supports the extravagant claim that infinities exist - infinite numbers of universes hosting countless galaxies. This leads to wellknown paradoxes, such as the infinite repetition of everything because of the finiteness of possibilities. But again, there is no way to test it, because infinity is always beyond reach - and so will not plausibly exist in physical reality, as mathematician David Hilbert argued.

The gap in current theories that warrants pursuing such untestable theories is our inability to predict firmly why physical constants have the values they do. If a fundamental theory were to be proposed that explained them, the drive for a multiverse explanation would fall away. But the puzzle of why these values allow life would remain.

The multiverse argument is a well-founded philosophical proposal but, as it cannot be tested, it does not belong fully in the scientific fold. Read The Hidden Reality with enjoyment, but beware its misleading title. Greene is not presenting aspects of a known reality; he is telling of unproven theoretical possibilities.

George Ellis is professor emeritus of applied mathematics at the University of Cape Town, Rondebosch 7701, South Africa. e-mail:george.ellis@uct.ac.za

\section{Books in brief}

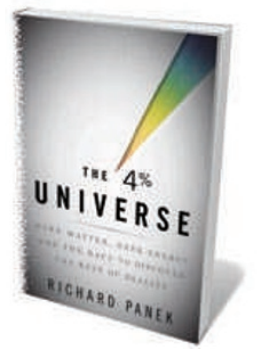

The 4\% Universe: Dark Matter, Dark Energy, and the Race to Discover the Rest of Reality

Richard Panek HOUGHTON MIFfLIN HARCOURT 320 pp. \$26 (2011)

The more cosmologists have learned in recent decades, the less they seem to know about the Universe. Just 4\% of it is composed of normal matter, made of protons and neutrons. The rest is 'dark matter' of unknown origin, and 'dark energy', a force that pulls space apart. Science writer Richard Panek engagingly tells the story of the discovery of these cosmic ingredients through interviews with the astronomers who unearthed them, charting the often-bitter rivalries between competing researchers.

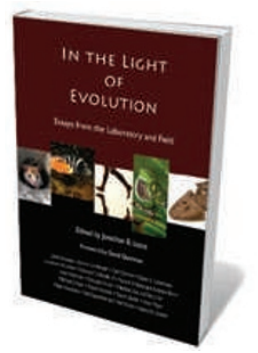

In the Light of Evolution: Essays from the Laboratory and Field Edited by Jonathan B. Losos ROBERTS AND COMPANY PUBLISHERS 336 pp. $\$ 49.95$ (2011)

Leading experts in evolution report from the field in this collection of plainspoken essays, providing a valuable resource for nonspecialists wanting to improve their understanding of this vital topic. Historian Janet Browne writes on Charles Darwin; writer Carl Zimmer muses on microbes; Daniel Lieberman discusses the evolution of human bipedalism; Marlene Zuk and Teri Orr examine sexual selection; and Neil Shubin unearths tetrapods and evolutionary steps in the fossil record.

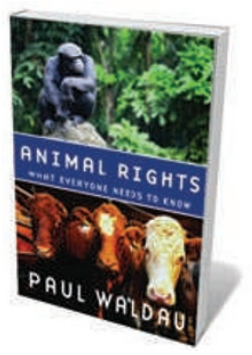

Animal Rights: What Everyone Needs to Know Paul Waldau OXFORD UNIVERSITY PRESS 256 pp. \$16.95 (2011) Animal-rights theory has a long history, as legal scholar Paul Waldau describes in this primer. Setting out the basics of animal protection law, he explains our different attitudes to pets and wild animals, research and work animals, and the creatures we eat. He chronicles how our understanding of animal welfare has developed and how it has led to protective measures and legislation as well as a passionate — and often controversial — animal-rights movement. He ends with proposals for a more unified framework for animal rights.

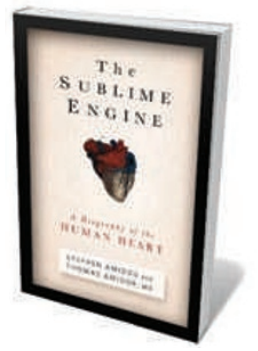

The Sublime Engine: A Biography of the Human Heart Stephen Amidon and Thomas Amidon RODALE BOOKS 224 pp. $\$ 24.99$ (2011)

The human heart is a fundamental organ on which we rely for our life force. For millennia, it has also been imbued with symbolism across many cultures. In their biography of the heart, writer Stephen Amidon and cardiologist Thomas Amidon trace the influence of the body's central pump through history, science, religion, literature and popular culture. From ancient Egypt and Greece, through the Middle Ages to the modern era, they ponder the miracle that is encased within our ribs.

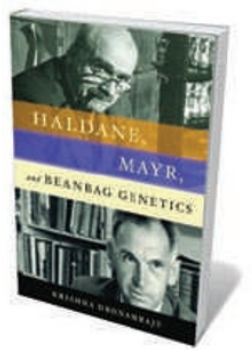

Haldane, Mayr, and Beanbag Genetics

Krishna Dronamraju OXFORD UNIVERSITY PRESS 296 pp. \$34.95 (2011) In the mid-twentieth century, two great biologists - J. B. S. Haldane and Ernst Mayr — clashed about the value of mathematical theories to evolution. Mayr, in 1959, queried Haldane's 'beanbag' approach to genetics, which portrayed evolution merely as "the adding of certain beans to a beanbag and the withdrawing of others". Haldane refuted Mayr's position in a witty essay in 1964. Geneticist Krishna Dronamraju relates their vigorous exchange through the scientists' correspondence. 